\title{
Exposure to Mebendazole and Pyrvinium during Pregnancy: A Danish Nationwide Cohort Study
}

\author{
A. Torp-Pedersen, ${ }^{1,2}$ E. Jimenez-Solem, ${ }^{1,2}$ J. T. Andersen, ${ }^{1,2}$ \\ K. Broedbaek, ${ }^{1,2}$ C. Torp-Pedersen, ${ }^{3}$ and H. E. Poulsen ${ }^{1,2}$ \\ ${ }^{1}$ Department of Clinical Pharmacology, Bispebjerg Hospital, Copenhagen, Denmark \\ ${ }^{2}$ Laboratory of Clinical Pharmacology Q7642, Rigshospitalet, Copenhagen, Denmark \\ ${ }^{3}$ Department of Cardiology, Gentofte Hospital, Denmark
}

Correspondence should be addressed to A. Torp-Pedersen, arendse.laerke.torppedersen@rh.regionh.dk

Received 29 June 2012; Accepted 21 August 2012

Academic Editor: Bryan Larsen

Copyright (C) 2012 A. Torp-Pedersen et al. This is an open access article distributed under the Creative Commons Attribution License, which permits unrestricted use, distribution, and reproduction in any medium, provided the original work is properly cited.

Purpose. Families with children are frequently exposed to pinworm infection and treatment involves the whole family. Information on consequences of exposure during, pregnancy is limited. The aim of this study was to investigate the exposure to pyrvinium and mebendazole before, during, and after pregnancy in a Danish nationwide cohort. Methods. From nationwide administrative registers, we identified 718, 900 births in Denmark between January 1997 and December 2007 as well as maternal prescription data of anthelmintics and maternal characteristics. Redemption of a prescription for pyrvinium or mebendazole was used to identify exposure. Results. 4715 women redeemed a prescription for pyrvinium or mebendazole during pregnancy; 1606 for pyrvinium, 2575 for mebendazole, and 534 for both drugs. Having $>2$ children compared to having no previous children was associated with exposure to pyrvinium (OR: 7.1, 95\% CI: 5.8-8.7) and mebendazole (OR: 20.8, 95\% CI: 17.3-24.9). Conclusion. 4715 pregnant women redeemed a prescription for either mebendazole or pyrvinium. We believe the exposure to be even higher since pyrvinium is also sold over-the-counter. Limited information on birth outcomes is available at present time, and considering the number of exposed pregnancies, we recommend that studies are to be undertaken to assess the safety of pyrvinium and mebendazole during pregnancy.

\section{Introduction}

In Denmark, mebendazole and pyrvinium are used in the treatment of enterobiasis caused by enterobius vermicularis (threadworm, seatworm, or pinworm).

The pinworm is a nematode that mostly infects children. In Denmark, the prevalence among children has been reported to be $29 \%$ [1]. Among pregnant women the prevalence is unknown.

Pinworm infections spread easily between family members. Treatment of the whole family is therefore recommended, if a child has been infected. Pinworms are commonly resided in the colon, but can in rare cases migrate to the vagina or bladder and cause a variety of symptoms such as vaginitis, cervicitis, endometritis, myometritis, and salpingitis [2]. Most pinworm infections are, however, symptom-free and harmless.

Mebendazole is the drug of choice for treating pinworm infections outside pregnancy. During pregnancy the recommended treatment in Denmark is pyrvinium due to its low systemic absorption [3]. Treatment of nematodes in pregnancy has been shown to reduce early infant mortality [4]. There is, however, limited information concerning the frequency of use of these drugs during pregnancy, and whether these recommendations are followed.

Studies in pregnant women have not shown negative birth outcomes associated with exposure to mebendazole [5-8]. We found no studies exploring birth outcomes after exposure to pyrvinium during pregnancy. Both drugs have a low absorption level in the intestine, which suggests that they 
are safe to use during pregnancy. However, both drugs are labelled as category $\mathrm{C}$, which indicates that more information is needed to ascertain their safety during pregnancy.

In order to estimate possible public health consequences of prenatal exposure, it is important to know the number of pregnant women being treated with mebendazole or pyrvinium.

The aim of this study is therefore to investigate the exposure to pyrvinium and mebendazole during pregnancy in a nationwide cohort.

\section{Subjects and Methods}

Our study cohort includes all live births in Denmark between 1997 and 2007. All subjects and information about them were gathered from three Danish national registries and Statistics Denmark [9]: The Danish Fertility Database [10], The Danish National Hospital Register [11], and The Danish National Prescription Register [12]. From these registers and Statistics Denmark, information at individual level was gathered via the unique personal identification number given to all Danish residents at birth or upon immigration [13].

From The Danish Fertility Database we identified all births in Denmark from January 1st, 1997 to December 31st, 2007 and the personal id numbers of the respective mothers. We also gathered information about maternal parity, date of conception, and date of delivery from this database. The date of conception is based on ultrasound estimates and information on date of last menstrual period. Gestational age was defined as starting on the day of conception, which was defined as the first day of the last menstrual period plus 14 days. This definition of gestational age should not be confused with the "classical gestational age" ranging from the first day of the last menstrual period until birth.

The Danish National Hospital Register has since 1977 included individual level data on all discharges from Danish hospitals, and since 1994 also individual level data on all outpatient visits [11]. From this register we acquired the pregnant mothers smoking habits according to the international classification of diseases (ICD-10, Danish revision), codes DUT00-99.

From two subsets held by Statistics Denmark (The Population's Education Register and The Income Statistics Register) we identified the mothers' highest level of completed education and their annual household income at birth year.

The Danish National Prescription Register contains information on all redeemed prescriptions from Danish pharmacies since 1995 [12]. We identified all prescriptions redeemed by the mothers in the study period via their personal id number. To identify exposure we used the Anatomical Therapeutic Chemical (ATC) classification; P02CX01 for pyrvinium and P02CA01 for mebendazole [14].

Pyrvinium is also available over-the-counter in Danish pharmacies; why an estimation of the redeemed prescription for pyrvinium could underestimate the true exposure.

2.1. Exposure. The date of redemption of a prescription for mebendazole or pyrvinium was used to identify exposure, which was classified according to first, second, and third trimester. First trimester was defined as the period between day of conception and day 84 of pregnancy, second trimester between day 85 and 196 of pregnancy, and third trimester between day 197 of pregnancy, and birth.

Cases were divided into three groups: exposed to pyrvinium, exposed to mebendazole, and exposed to both pyrvinium and mebendazole.

Treatment against pinworm infections consists of two doses given two weeks apart to prevent reinfection. The second treatment may therefore extend into the following trimester.

To analyze differences in patterns of redemption before and after pregnancy, we furthermore identified women exposed to pyrvinium and mebendazole $0-3$ and 6-9 months before and after pregnancy.

To assess the development of exposure to pyrvinium or mebendazole over the years we studied the incidence for each year in the study period.

2.2. Statistics. Maternal characteristics (age, parity, smoking, income, and education) are presented as frequencies with percentages. Differences between categorical variables for the exposed versus unexposed were assessed by Chi-square $\left(\chi^{2}\right)$ tests. We considered two-sided $P$ values $<0.05$ to be statistically significant. To estimate if maternal characteristics were associated with being exposed to either pyrvinium or mebendazole we performed multivariable logistic regressions with odds ratios (OR) and 95\% confidence intervals (CI) as results. We adjusted for age, parity, smoking, income, and education.

We classified five age groups for maternal age at conception: $<20,20-24,25-29,30-35$, and $>35$ years $(0 \%$ missing data). Parity was defined as the number of previous live births, and classified into four groups: $0,1,2$, and $>2$ birth(s) $(<1 \%$ missing data).

We classified highest level of completed education into three groups: low, medium, and high (4.0\% missing data). Annual household income during the year of birth was classified into quartiles ( $<1 \%$ missing data). Finally, smoking was classified into smokers and nonsmokers $(6.8 \%$ missing data). Statistical analyses and data management were all performed using SAS 9.2 (SAS Institute Inc., Cary, NC, USA).

2.3. Ethics. Ethical approval is not required for register-based studies in Denmark. All personal information held in the registers was encrypted and analyzed on computers held by Statistics Denmark. The study has been approved by The Danish Data Protection Agency (no. 2008-41-2517).

\section{Results}

We identified 759, 690 births between 1997 and 2007. We excluded 117 records due to coding errors, 17 duplicate records, and 40675 records with missing data on gestational age. Our final study population consisted of 718, 900 births $-94.63 \%$ of all recorded births. 


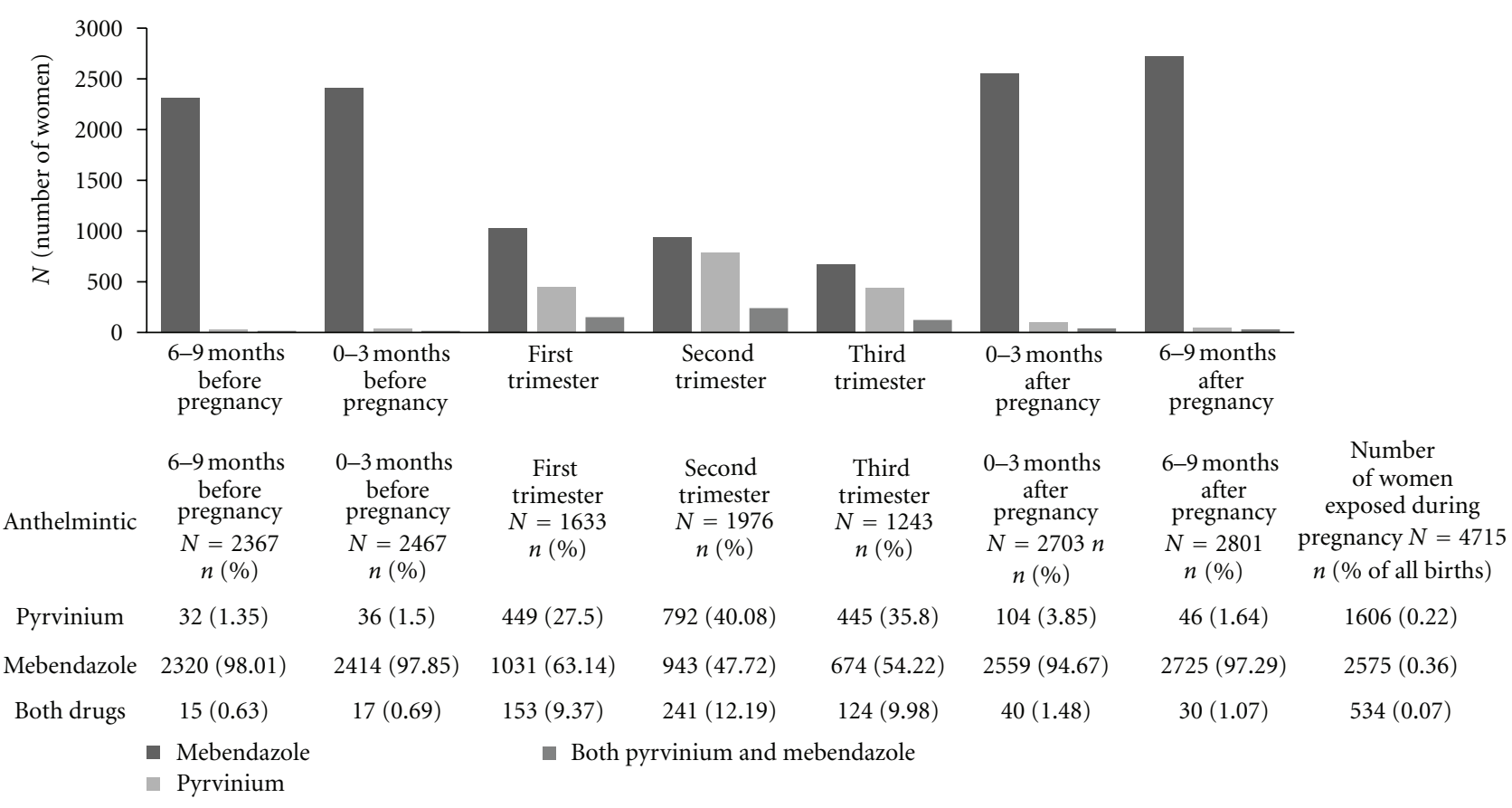

Figure 1: The maternal exposure to pyrvinium, mebendazole, and both drugs before, during, and after pregnancy, expressed by seven three-month time frames. For a better overview the data is shown in tables and figures.

3.1. Exposure. We identified 4715 (0.65\%) mothers redeeming a prescription for pyrvinium or mebendazole during pregnancy; $1606(0.22 \%)$ for pyrvinium, 2575 (0.36\%) for mebendazole, and 534 (0.07\%) for both drugs (Figure 1).

Of the 1606 women redeeming a prescription for pyrvinium, 245 redeemed the drug twice, and 39 redeemed it more than twice during pregnancy.

Of the 2575 women redeeming a prescription for mebendazole, 508 redeemed the drug twice, and 31 redeemed it more than twice during pregnancy.

The exposure over the three trimesters was as follows.

(i) Pyrvinium: 449 pregnancies in the first trimester, of which 434 were exposed from day 14 to 84 after conception, which is believed to be the most vulnerable period for congenital malformations, 792 pregnancies in the second trimester, and 445 pregnancies in the third trimester.

(ii) Mebendazole: 1031 pregnancies in first the trimester, of which 694 were exposed from day 14 to 84 after conception, 943 pregnancies in the second trimester, and 674 pregnancies in the third trimester.

(iii) Mebendazole and pyrvinium: 153 pregnancies in the first trimester, of which 139 were exposed from day 14 to 84 after conception, 241 pregnancies in the second trimester, and 124 pregnancies in the third trimester.

The number of women redeeming a prescription for a drug 0-3 months before pregnancy was 36 for pyrvinium, 2414 for mebendazole, and 17 for both drugs. The number of women redeeming a prescription for a drug 0-3 months after pregnancy was 104 for pyrvinium, 2559 for mebendazole, and 40 for both drugs.
The number of women redeeming a prescription for a drug 6-9 months before pregnancy was 32 for pyrvinium, 2320 for mebendazole, and 15 for both drugs. The number of women redeeming a prescription for a drug 6-9 months after pregnancy was 45 for pyrvinium, 2725 for mebendazole, and 30 for both drugs.

We compared the number of women redeeming a prescription in the three months before pregnancy with the first trimester and found a significant increase in redeemed prescriptions for pyrvinium $(P<0.0001)$ and a significant decrease in the number of women redeeming a prescription for mebendazole $(P<0.0001)$. When comparing the number of women redeeming a prescription in the third trimester with the three months after birth, we found a significant decrease in the number of women redeeming a prescription for pyrvinium $(P<0.0001)$ and a significant increase in number of women redeeming a prescription for mebendazole $(P<0.0001)$.

The percentage of pregnant women redeeming a prescription for pyrvinium or mebendazole in the study period (1997-2007) varied between $0.20 \%$ and $0.25 \%$ for pyrvinium, $0.26 \%$ and $0.56 \%$ for mebendazole, and $0.04 \%$ and $0.09 \%$ for both drugs (Figure 2).

3.2. Maternal Characteristics. Characteristics of exposed and unexposed women are shown in Table 1. Women exposed to pyrvinium, mebendazole, or both drugs during pregnancy were older $(P<0.0001)$ and had a higher parity $(P<0.0001)$ compared to unexposed.

Mebendazole-exposed women had a lower level of completed education $(P=0.002)$ but no significant difference in income $(P=0.25)$ compared to unexposed, while pyrvinium 


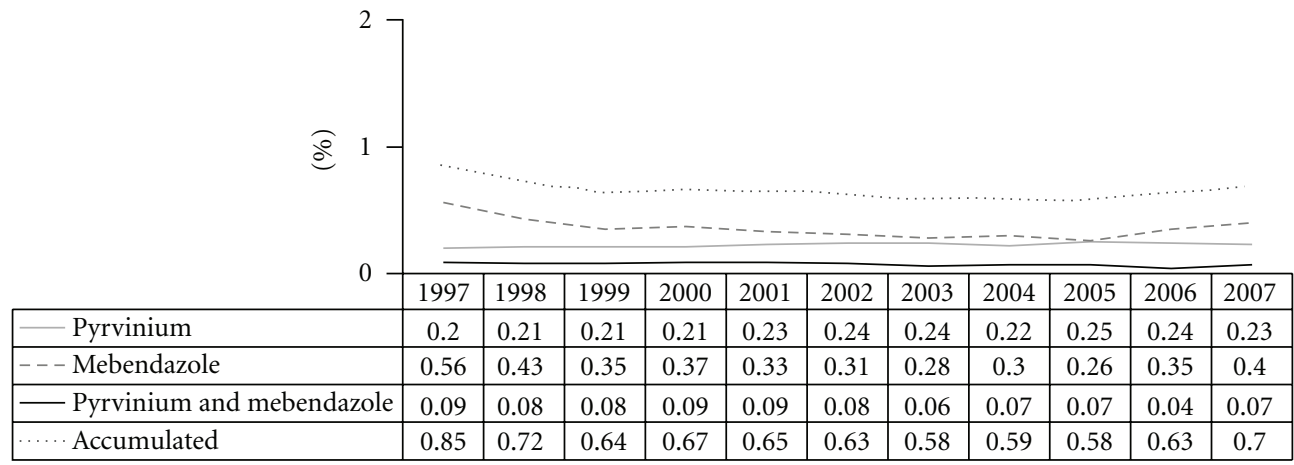

Figure 2: Percentage of pregnancies exposed to pyrvinium, mebendazole, and both drugs for each year in the study period (1997-2007). For each year, data is presented as percentages of all births in the corresponding year.

TABLE 1: Characteristics of exposed and unexposed women.

\begin{tabular}{|c|c|c|c|c|}
\hline Drug exposure & Pyrvinium $n(\%)$ & Mebendazole $n(\%)$ & Both drugs $n(\%)$ & Unexposed $n(\%)$ \\
\hline \multicolumn{5}{|l|}{ Age } \\
\hline$<20$ & $33(2.5)$ & $28(1.1)$ & $4(0.8)$ & $19649(2.8)$ \\
\hline $20-24$ & $178(11.1)$ & $209(8.1)$ & $43(8.1)$ & $111765(15.7)$ \\
\hline $25-29$ & $501(31.2)$ & $772(30.0)$ & $147(27.5)$ & $274107(38.4)$ \\
\hline $30-34$ & $637(39.7)$ & $1069(41.5)$ & $230(43.1)$ & $224422(31.4)$ \\
\hline$>35$ & $257(16.0)$ & $497(19.3)$ & $110(20.6)$ & $84242(11.8)$ \\
\hline$P$ value* & $P<0.0001$ & $P<0.0001$ & $P<0.0001$ & \\
\hline \multicolumn{5}{|l|}{ Income } \\
\hline Quartile 1 & $398(24.8)$ & $655(25.4)$ & $119(22.3)$ & $178476(25.0)$ \\
\hline Quartile 2 & $385(24.0)$ & $680(26.4)$ & $117(21.9)$ & $178466(25.0)$ \\
\hline Quartile 3 & $428(26.7)$ & $614(23.8)$ & $140(26.2)$ & $178466(25.0)$ \\
\hline Quartile 4 & $395(24.6)$ & $877(24.3)$ & $158(29.6)$ & $178470(25.0)$ \\
\hline$P$ value* & $P=0.47$ & $P=0.25$ & $P=0.04$ & \\
\hline \multicolumn{5}{|l|}{ Parity } \\
\hline 0 & $309(19.2)$ & $229(8.9)$ & $55(10.3)$ & $310740(43.5)$ \\
\hline 1 & $574(35.7)$ & $860(33.4)$ & $172(32.2)$ & $266754(37.4)$ \\
\hline 2 & $499(31.1)$ & $959(37.2)$ & $209(39.1)$ & $100523(14.1)$ \\
\hline$>2$ & $224(14.0)$ & $527(20.5)$ & $98(18.4)$ & $36152(5.1)$ \\
\hline$P$ value* & $P<0.0001$ & $P<0.0001$ & $P<0.0001$ & \\
\hline \multicolumn{5}{|l|}{ Education class } \\
\hline Low & $566(35.8)$ & $908(35.8)$ & $176(33.3)$ & $241803(35.3)$ \\
\hline Medium & $492(31.1)$ & $747(29.4)$ & $144(27.3)$ & $222398(32.4)$ \\
\hline High & $522(33.0)$ & $883(34.8)$ & $208(39.4)$ & $221316(32.3)$ \\
\hline$P$ value* & $P=0.54$ & $P=0.002$ & $P=0.001$ & \\
\hline \multicolumn{5}{|l|}{ Smoking } \\
\hline Yes & $266(18.0)$ & $435(18.3)$ & $91(18.4)$ & $121178(18.2)$ \\
\hline No & $1215(82.0)$ & $1948(81.8)$ & 405 (81.7) & $544192(81.8)$ \\
\hline$P$ value* & $P=0.80$ & $P=0.96$ & $P=0.94$ & \\
\hline
\end{tabular}

*The $P$ values are results of $\chi^{2}$ tests comparing each exposure group with the unexposed group.

exposed did not differ in level of education $(P=0.54)$ or income $(P=0.47)$ compared to unexposed. Women who had been exposed to both drugs during pregnancy had a higher level of education $(P=0.001)$ and a higher income $(P=0.04)$ compared to unexposed women.

We found no difference in the proportion of smokers in any of the exposed groups compared to the unexposed group $(P=0.80,0.96$, and 0.94$)$.
Predictors of exposure are shown in Table 2. Having a higher parity was associated with being exposed to mebendazole or pyrvinium. Having more than 2 children compared to no children resulted in an OR $=7.07$ (95\% CI 5.75-8.68) for exposure to pyrvinium during pregnancy and an $\mathrm{OR}=$ 20.76 (95\% CI 17.31-24.88) for exposure to mebendazole during pregnancy when compared to being unexposed. Having two children compared to no children resulted in 
TABle 2: Predictors of exposure to pyrvinium and mebendazole during pregnancy expressed by odds ratios (OR) and $95 \%$ confidence intervals (CI).

\begin{tabular}{lcc}
\hline & Pyrvinium versus unexposed OR* $(95 \%$ CI $)$ & Mebendazole versus unexposed OR* (95\% CI) \\
\hline Age & $0.72(0.47-1.10)$ & $0.74(0.48-1.16)$ \\
$20-24$ versus $<20$ & $0.65(0.42-0.98)$ & $0.74(0.48-1.15)$ \\
$25-29$ versus $<20$ & $0.71(0.47-1.10)$ & $0.77(0.50-1.20)$ \\
$30-34$ versus $<20$ & $0.61(0.39-0.95)$ & $0.69(0.44-1.08)$ \\
$>35$ versus $<20$ & & $1.15(1.03-1.30)$ \\
\hline Income & $1.03(0.88-1.19)$ & $1.01(0.89-1.14)$ \\
Quartile 2 versus 1 & $1.14(0.98-1.33)$ & $0.95(0.84-1.08)$ \\
Quartile 3 versus 1 & $0.99(0.84-1.16)$ & $4.30(3.67-5.04)$ \\
Quartile 4 versus 1 & & $12.70(10.79-14.94)$ \\
\hline Parity & $2.31(1.98-2.69)$ & $20.76(17.31-24.88)$ \\
1 versus 0 & $5.38(4.56-6.35)$ & $1.02(0.92-1.13)$ \\
2 versus 0 & $7.07(5.75-8.68)$ & $1.28(1.15-1.44)$ \\
$>2$ versus 0 & & $0.92(0.82-1.02)$ \\
\hline Education & $1.01(0.89-1.16)$ & \\
$\quad$ Medium versus low & $1.08(0.94-1.25)$ & \\
$\quad$ High versus low & &
\end{tabular}

* Adjusted for age, income, parity, level of completed education, and smoking.

an $\mathrm{OR}=5.38$ (95\% CI 4.56-6.35) for exposure to pyrvinium during pregnancy and an $\mathrm{OR}=12.70$ (95\% CI 10.79 14.94) for exposure to mebendazole during pregnancy when compared to being unexposed. Having one child compared to no children resulted in an $\mathrm{OR}=2.31$ (95\% CI 1.98-2.69) for exposure to pyrvinium and an OR $=4.30$ (95\% CI 3.675.04) for exposure to mebendazole during pregnancy when compared to being unexposed.

\section{Discussion}

Among the 718, 900 births in our study cohort, $0.65 \%$ of the mothers redeemed prescriptions for pyrvinium, mebendazole, or both drugs during pregnancy. This incidence appears to have been steady between 1997 and 2007 (Figure 2).

We observed a shift towards using pyrvinium during pregnancy, which reflects the recommendation to prefer this drug in pregnancy (Figure 1). Even so mebendazole is still the more redeemed drug.

As could be expected, having a higher parity was associated with being exposed to pyrvinium and mebendazole. The most likely explanation is that having a higher number of children increases the risk of them being infected with pinworms. The number of siblings in a household has previously been linked with increasing risk of pinworm infections $[15,16]$.

We found no other studies exploring the frequency of exposure to pyrvinium or mebendazole in pregnant women. The prevalence of pinworm infections in children has been reported to be similar in Denmark as in Sweden [17], Norway [15], and Estonia [16]. Therefore, we believe that the proportion of pregnant women exposed to anthelmintics may be similar in these countries.
4.1. Strengths and Limitations. This Danish nationwide cohort study includes information on all births and the mothers' redemption of prescriptions of pyrvinium and mebendazole in the study period. Information from the Danish national registers used in this study was obtained prospectively and not based on questionnaires or interviews. Recall bias is therefore eliminated.

Danish pharmacies are obliged to register all redeemed prescriptions as part of the national health care reimbursement scheme. As a result, The Danish National Prescription Register includes approximately $97.5 \%$ of all redeemed prescriptions in Denmark [18].

The accuracy of our results, however, depends on the association between the redemption of prescriptions for pyrvinium or mebendazole and actual drug ingestion, since the redemptions of prescriptions for pyrvinium or mebendazole are used as proxy measures for exposure to the drugs. Lack of compliance would lead to misclassifying unexposed women to be exposed, which would overestimate the exposure. The exposure to pyrvinium and mebendazole is based on information on redeemed and paid prescriptions, which increases the probability of exposure. Furthermore, if not all family members are treated, reinfection might occur. Women may be more prone to receive treatment to avoid a possible risk of reinfection.

A major limitation in this study is the underestimated exposure to pyrvinium, since pyrvinium is also available over-the-counter in Danish pharmacies. Information from the Danish Medicines Agency on the total amount of pyrvinium purchased in pharmacies in the years 1999-2007 states that only $4-7 \%$ of all sales was by the use of a prescription [19]. This percentage may be different among pregnant women, but surely indicates that the true exposure to 
pyrvinium among pregnant women is higher than that found in this study.

\section{Conclusion}

4715 Danish pregnant women redeemed a prescription for either mebendazole or pyrvinium. We believe this number to be even higher since pyrvinium is also sold over-the-counter. Limited information on birth outcomes is available at present time, and considering the number of exposed pregnancies, we recommend that studies are to be undertaken to assess the safety of pyrvinium and mebendazole during pregnancy.

\section{Conflict of Interests}

The authors declare that they have no conflict of interests.

\section{References}

[1] M. Lacroix and B. Sørensen, "Occurrence of Enterobius vermicularis in children hospitalized at a central hospital," Ugeskrift for Laeger, vol. 162, no. 9, pp. 1236-1238, 2000.

[2] V. St Georgiev, "Chemotherapy of enterobiasis (oxyuriasis)," Expert Opinion on Pharmacotherapy, vol. 2, no. 2, pp. 267-275, 2001.

[3] T. C. Smith, A. W. Kinkel, C. M. Gryczko, and J. R. Goulet, "Absorption of pyrvinium pamoate," Clinical Pharmacology and Therapeutics, vol. 19, no. 6, pp. 802-806, 1976.

[4] P. Christian, S. K. Khatry, and P. K. P. West Jr., "Antenatal anthelmintic treatment, birthweight, and infant survival in rural Nepal," The Lancet, vol. 364, no. 9438, pp. 981-983, 2004.

[5] N. R. de Silva, J. L. G. J. Sirisena, D. P. S. Gunasekera, M. M. Ismail, and H. J. de Silva, "Effect of mebendazole therapy during pregnancy on birth outcome," The Lancet, vol. 353, no. 9159, pp. 1145-1149, 1999.

[6] G. G. Briggs, R. K. Freeman, and S. J. Yaffe, Eds., Drugs in Pregnancy and Lactation: A Reference Guide to Fetal and Neonatal Risk, Lippincott Williams \& Wilkins, Philadelphia, Pa, USA, 8th edition, 2008.

[7] O. Diav-Citrin, S. Shechtman, J. Arnon, I. Lubart, and A. Ornoy, "Pregnancy outcome after gestational exposure to mebendazole: a prospective controlled cohort study," American Journal of Obstetrics and Gynecology, vol. 188, no. 1, pp. 282-285, 2003.

[8] N. Ács, F. Bánhidy, E. Puhó, and A. E. Czeizel, "Populationbased case-control study of mebendazole in pregnant women for birth outcomes," Congenital Anomalies, vol. 45, no. 3, pp. 85-88, 2005.

[9] “Statistics Denmark," http://www.dst.dk/en.aspx.

[10] L. B. Knudsen, “The danish fertility database," Danish Medical Bulletin, vol. 45, no. 2, pp. 221-225, 1998.

[11] E. Lynge, J. L. Sandegaard, and M. Rebolj, "The Danish national patient register," Scandinavian Journal of Public Health, vol. 39, no. 7, pp. 30-33, 2011.

[12] H. W. Kildemoes, H. T. Sørensen, and J. Hallas, "The Danish national prescription registry," Scandinavian Journal of Public Health, vol. 39, no. 7, pp. 38-41, 2011.

[13] C. B. Pedersen, "The Danish civil registration system," Scandinavian Journal of Public Health, vol. 39, no. 7, pp. 22-25, 2011.

[14] ATC, "Structure and principles," http://www.whocc.no/atc/ structure_and_principles/.
[15] H. Boas, G. Tapia, J. A. Sødahl, T. Rasmussen, and K. S. Rønningen, "Enterobius vermicularis and risk factors in healthy Norwegian children," Pediatric Infectious Disease Journal, vol. 31, no. 9, pp. 927-930, 2012.

[16] M. Remm, "Distribution of enterobiasis among nursery school children in SE Estonia and of other helminthiases in Estonia," Parasitology Research, vol. 99, no. 6, pp. 729-736, 2006.

[17] P. Herrström, A. Friström, A. Karlsson, and B. Högstedt, "Enterobius vermicularis and finger sucking in young Swedish children," Scandinavian Journal of Primary Health Care, vol. 15, no. 3, pp. 146-148, 1997.

[18] H. T. Sørensen, I. Hansen, E. Ejlersen, S. Sabroe, and H. Hamburger, "Identification of patients treated with strong analgesics: an assessment of two Danish information systems with respect to epidemiological research," Journal of Medical Systems, vol. 20, no. 1, pp. 57-65, 1996.

[19] Medstat, http://www.medstat.dk/. 


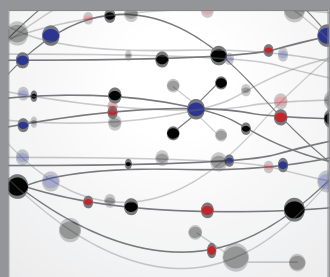

The Scientific World Journal
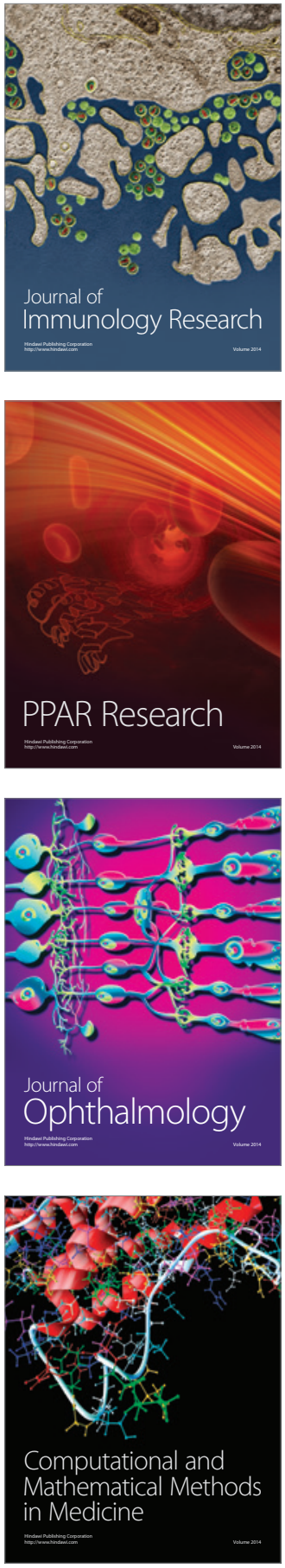

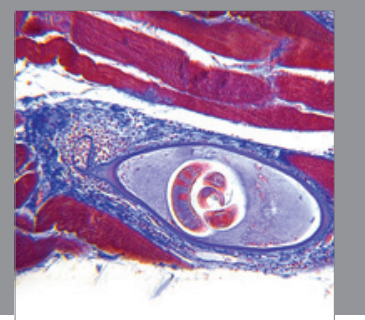

Gastroenterology

Research and Practice
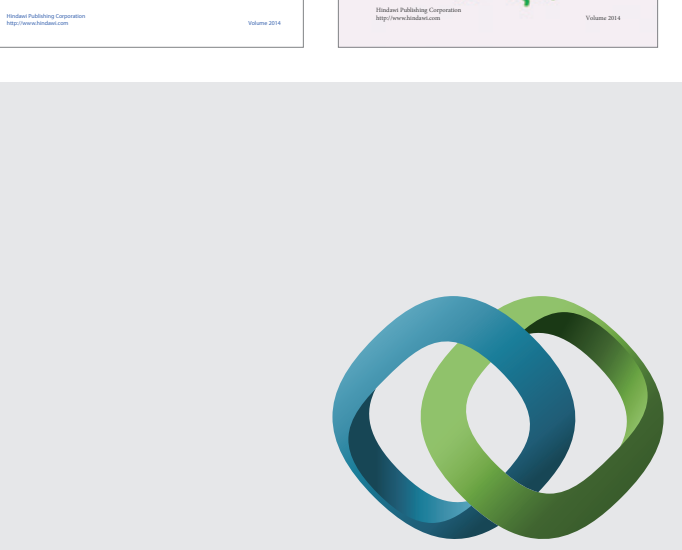

\section{Hindawi}

Submit your manuscripts at

http://www.hindawi.com
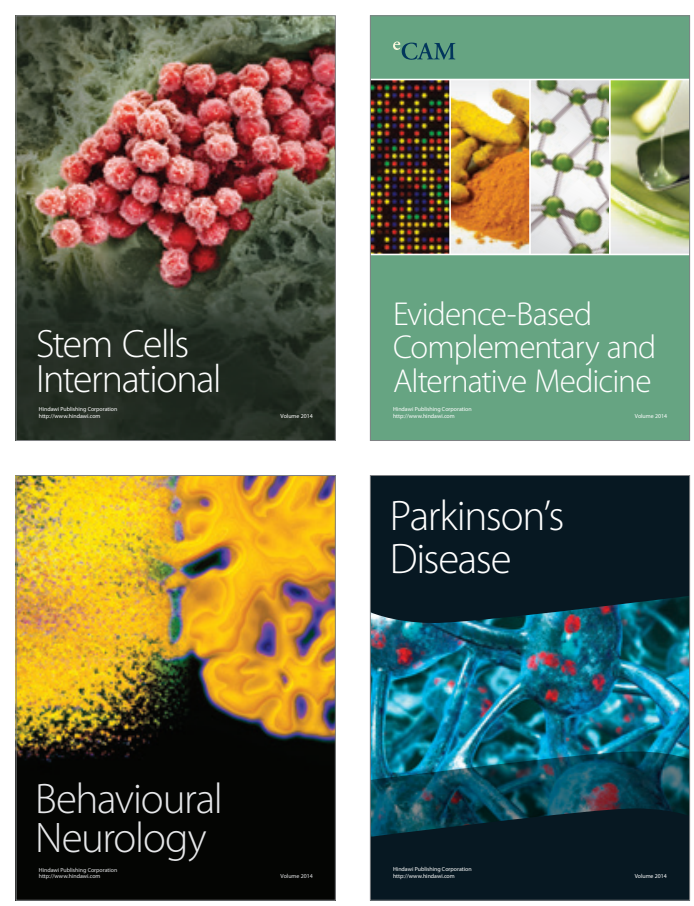

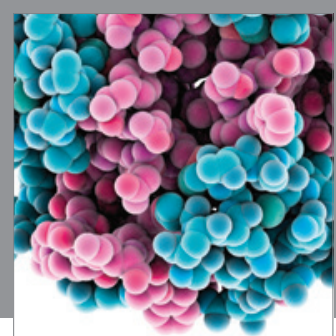

Journal of
Diabetes Research

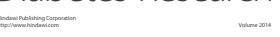

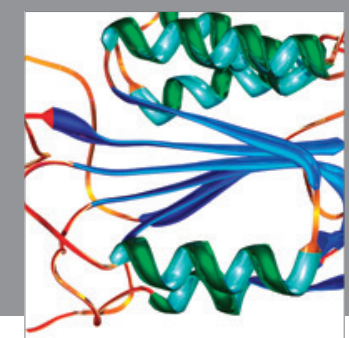

Disease Markers
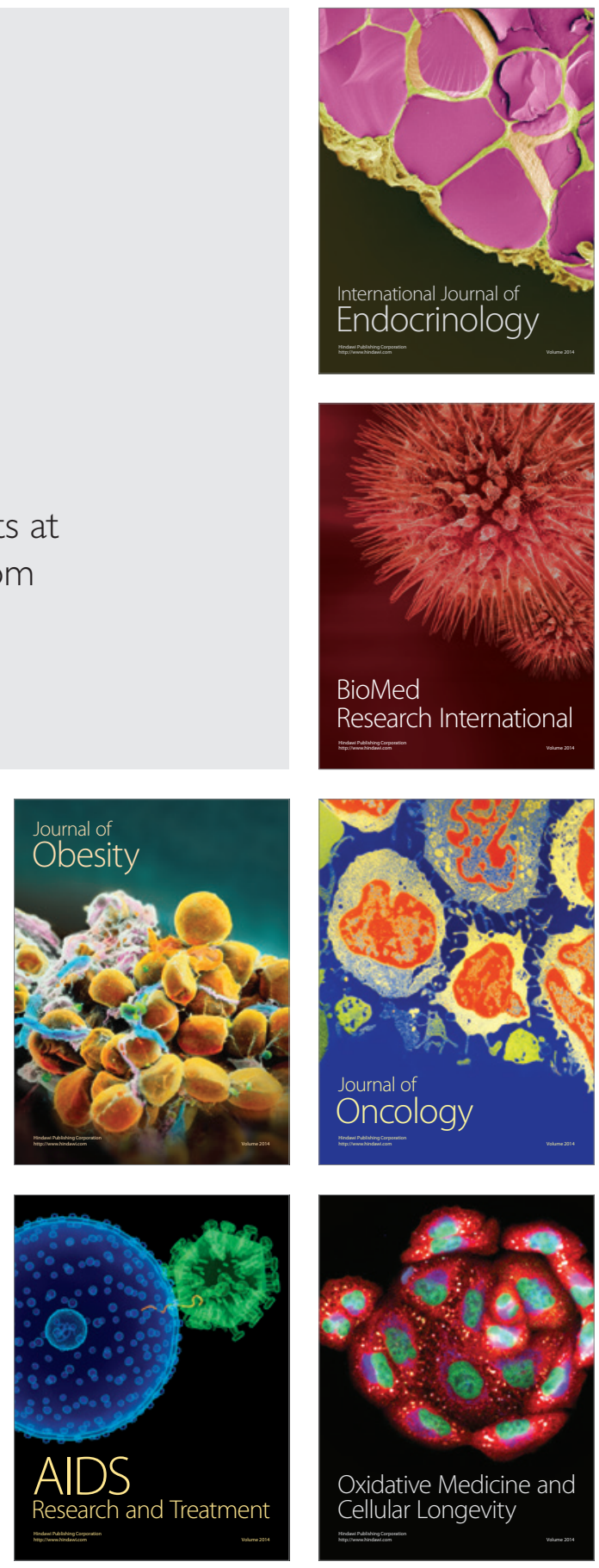